\title{
DEVELOPMENT OF THE ENIGMA FUEL PERFORMANCE CODE FOR WHOLE CORE ANALYSIS AND DRY STORAGE ASSESSMENTS
}

\author{
GLYN ROSSITER* \\ Fuel Cycle Solutions, UK National Nuclear Laboratory (NNL) \\ Springfields site, Salwick, Preston, Lancashire, PR4 0XJ, United Kingdom \\ *Corresponding author. E-mail : glyn.d.rossiter@nnl.co.uk
}

Received November 16, 2011

UK National Nuclear Laboratory's (NNL's) version of the ENIGMA fuel performance code is described, including details of the development history, the system modelled, the key assumptions, the thermo-mechanical solution scheme, and the various incorporated models. The recent development of ENIGMA in the areas of whole core analysis and dry storage applications is then discussed. With respect to the former, the NEXUS code has been developed by NNL to automate whole core fuel performance modelling for an LWR core, using ENIGMA as the underlying fuel performance engine. NEXUS runs on NNL's GEMSTONE high performance computing cluster and utilises 3-D core power distribution data obtained from the output of Studsvik Scandpower's SIMULATE code. With respect to the latter, ENIGMA has been developed such that it can model the thermo-mechanical behaviour of a given LWR fuel rod during irradiation, pond cooling, drying, and dry storage — this involved: (a) incorporating an out-of-pile clad creep model for irradiated Zircaloy-4; (b) including the ability to simulate annealing out of the clad irradiation damage; (c) writing of additional post-irradiation output; (d) several other minor modifications to allow modelling of post-irradiation conditions.

KEYWORDS : Fuel Performance, ENIGMA

\section{INTRODUCTION}

This paper discusses the recent development of NNL's version of the ENIGMA fuel performance code, which has focussed on whole core analysis and dry storage applications. ENIGMA as a whole is first described.

\section{DESCRIPTION OF ENIGMA}

ENIGMA [1-6] is the primary UK computer code for thermal reactor fuel performance analysis. It calculates the thermo-mechanical behaviour of a light water reactor (LWR) or advanced gas-cooled reactor (AGR) fuel rod in both steady-state and transient conditions. Further discussion below pertains to NNL's version of the code for LWR applications (henceforward simply referred to as ENIGMA).

$\mathrm{UO}_{2}$, mixed oxide ( $\left.\mathrm{MOX}\right),(\mathrm{U}, \mathrm{Gd}) \mathrm{O}_{2}$ and yttriastabilised zirconia inert matrix fuel types, and Zircaloy-2, Zircaloy-4 and modern zirconium-based alloy clad types can all be modelled. Stainless steel cladding has also historically been simulated. ENIGMA is available under licence from NNL, or through Studsvik Scandpower, where it can be sub-licensed in conjunction with their Core Management System (CMS) code suite, which includes the CASMO [7] and SIMULATE [8] neutronics software.

ENIGMA is written in FORTRAN. The development version runs on a Windows PC platform, while end-user versions run on various Windows, Unix and Linux platforms. The development version includes a screen graphics capability whereby key calculated quantities (together with corresponding measured data where available) are plotted on the screen as the simulation proceeds, with results retained at the end of the run as Portable Network Graphics (PNG) files. An example of the screen graphics output is reproduced as Fig. 1 .

ENIGMA is validated against a large database of LWR fuel rod irradiations in both commercial and test reactors. In total, over 500 rod irradiations with burnups up to $90 \mathrm{MWd} / \mathrm{kgHM}$ are included in the validation database. The validation system is highly automated, facilitating re-validation after any source code changes.

ENIGMA has been used for several design and licensing assessments for both $\mathrm{UO}_{2}$ and MOX fuel, including licensing $\mathrm{UO}_{2}$ and gadolinia-doped $\mathrm{UO}_{2}$ in the 

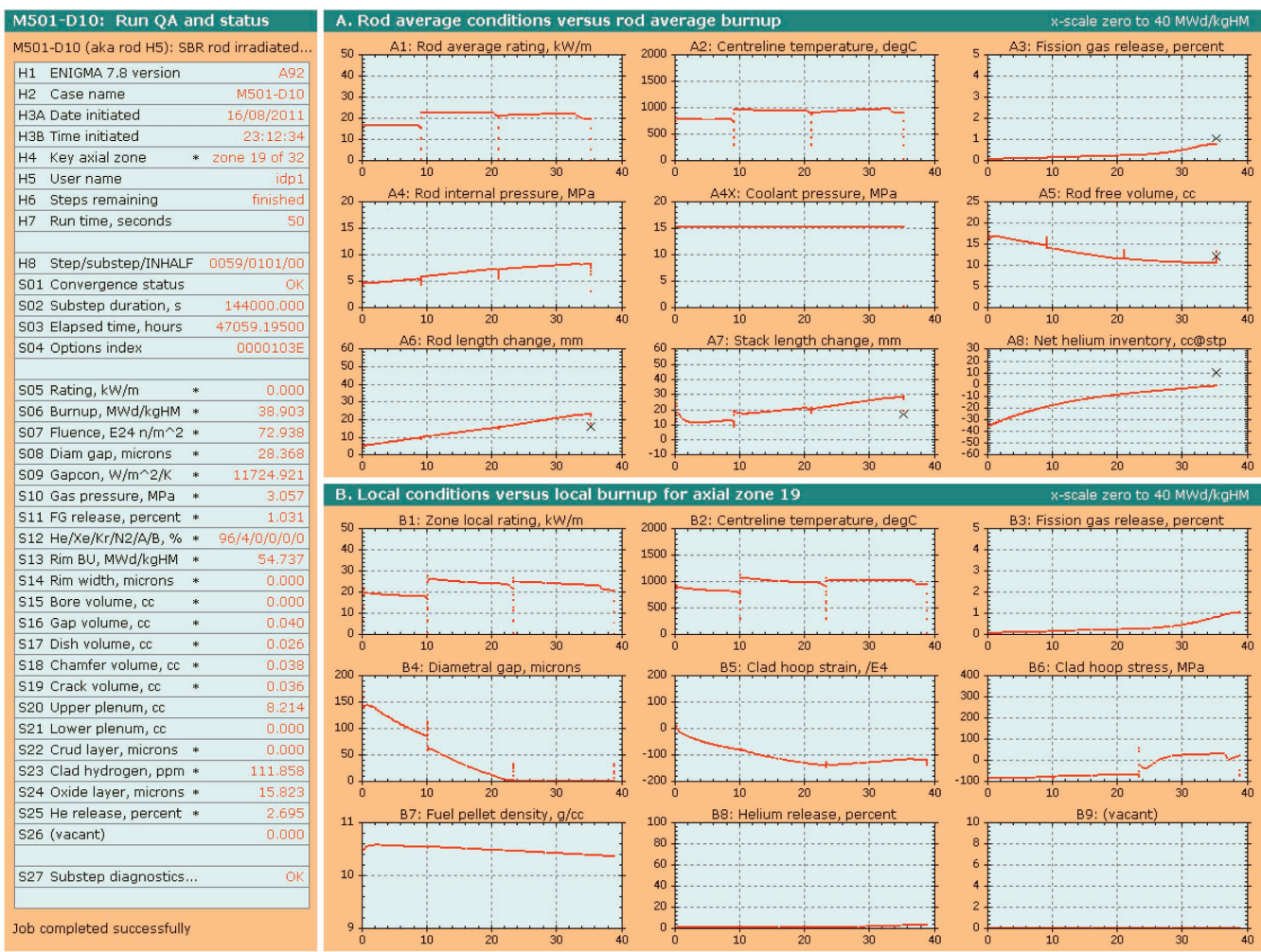

Fig. 1. Example of ENIGMA Screen Graphics Output (Red Lines Illustrate Code Predictions, while Blue Crosses show Measured Data)

UK Sizewell B PWR and the Finnish Loviisa VVER-440 reactor, and mixed oxide (MOX) fuel in the Swiss Beznau-1 PWR. ENIGMA is also used for analysis of experimental and commercial irradiations [9-15], for assessment of fuel behaviour during interim storage (see Section 4), to perform feasibility studies for future irradiation scenarios [16], to support fuel manufacturing $[11,14,15]$, and to investigate fuel failures or other fuel performance related problems [17].

\subsection{Development History}

The development of the ENIGMA code dates back to 1986, when work began at British Nuclear Fuels plc (BNFL) on the construction of a new modular code framework to act as a test bed for the development of sub-models. Around the same time, work was underway at the UK Central Electricity Generating Board (CEGB) on the creation of new models for a number of important properties and processes. The two strands came together in early 1988 when, with funding from the Sizewell B Project, a major programme of work was initiated to develop, document, verify and validate the code to a standard suitable for use in support of the Sizewell B PWR pre-operational safety report.

From this beginning, development of the code by both NNL (formerly the R\&D division of BNFL) and EDF
Energy (formerly CEGB) has continued, initially in collaboration, but since 1991 in parallel (BNFL ownership of ENIGMA transferred to NNL in 2005). While EDF Energy has developed the code for AGR use, NNL's developments have concentrated on LWR issues.

\subsection{System Modelled}

The active stack length region of the fuel rod is represented by a series of axial zones. In each axial zone the fuel is divided into radial annuli of equal thickness. This is the so-called $1^{1 / 2}$-D representation. The free volumes associated with the fuel-clad gap, pellet dishes and chamfers, pellet cracks, the pellet bore, and upper and lower plena are also modelled.

An isolated thermal-hydraulic subchannel can be simulated to calculate rod surface temperature boundary conditions where appropriate.

Out-of-pile conditions, rod re-fabrication, irradiation in two or more different reactors and the presence of fuel thermocouples can all be simulated.

\subsection{Key Assumptions}

Only radial, i.e. no axial or circumferential, heat flow is assumed and the fuel annuli are all considered to be subject to the same axial strain (the so-called plane strain 
assumption). The latter, in conjunction with an assumption of axi-symmetry, allows shear stresses to be ignored, such that only the normal stresses along the principal radial, circumferential and axial directions are non-zero. However: (a) the effects of shear stresses are approximated using models for axial extrusion and for pellet wheatsheafing which feed back calculated strain increments into the main solution scheme [5]; (b) the azimuthal cladding stress concentration over radial fuel cracks is calculated using a parasitic model [2]. Thus, the key phenomena which cannot be modelled with the 1-D plane axial strain assumption employed in the code's main solution scheme are instead modelled by other means.

Coupling between the axial zones is restricted to the coolant enthalpy, rod internal pressure and gas transport. Gas mixing in the free volume is assumed to be instantaneous.

Pellet cracking is approximately modelled by a directionally dependent fuel elastic modulus. The fuel radial elastic strains are calculated assuming the true elastic modulus. The fuel axial and circumferential elastic strains are calculated assuming the true elastic modulus if the corresponding stresses are compressive, but assuming a reduced elastic modulus if the stresses are tensile.

The cladding is treated as a membrane (or thin shell), i.e. thin wall equations are implemented.

\subsection{Solution Scheme}

The power history of each axial zone (heat generated, in $\mathrm{kW} / \mathrm{m}$, versus time) and thermal-hydraulic conditions at the bottom of the fuel stack are boundary conditions and are provided as input. Starting from the as-manufactured condition, ENIGMA then calculates the thermo-mechanical state of the fuel rod at the end of each timestep. A finite difference solution scheme is employed. The iteration strategy involves an inner loop to determine the temperature, stress and strain distributions for each axial zone using an iterative marching procedure, and an outer loop to feed in damped estimates of non-linear strain components (e.g. creep) and gas release. Both steady-state and transient heat transfer can be modelled.

If convergence fails during any particular timestep, the timestep length is halved and the step is automatically repeated. A stable solution is obtained, irrespective of timestep length, by calculating the stress and temperature distributions which, acting unchanged throughout the timestep, are compatible both with the imposed strains and with the fuel and clad stress-strain relationships.

The main calculations are performed at the pellet waist (or mid-pellet) elevation. The stresses and strains at the pellet end are then calculated parasitically.

\subsection{Models}

The models implemented for the fuel pellets include: thermal expansion; elasticity; heat capacity and enthalpy; radial power profile evolution [18]; thermal conductivity and its degradation with burnup; formation of high burnup structure at pellet rim; creep (both irradiation induced and thermal); densification (fuel temperature and grain size dependence); fuel matrix fission product swelling (including effects of solid, volatile and gaseous fission products - swelling is reduced when volatile and gaseous fission products accumulate in bubbles or are released from the fuel); axial extrusion and dish filling; pellet wheatsheafing; equi-axed grain growth (without grain boundary sweeping).

The models implemented for the cladding include: thermal expansion; elasticity; creep and instantaneous plasticity; axial growth; waterside corrosion and hydrogen pickup [38]; clad ridging (including stress concentration over radial fuel cracks) [2]; stress-corrosion crack growth; fatigue damage and ratchetting. Pellet-cladding interaction (PCI) is simulated assuming no sliding (no relative movement between pellets and cladding) in the axial direction, while a stick/slip situation is modelled azimuthally [2].

The models implemented for the various gases in the fuel rod include: fission gas generation (including the isotopics) [18]; (stable) fission gas release and gas bubble swelling; ${ }^{131}$ I generation and release; helium generation and release; helium adsorption and re-release; release of chemically absorbed nitrogen; instantaneous axial gas mixing; fuel-clad gap conductance (with conduction, radiation and empirical components - the empirical component takes account of pellet fragment relocation, pellet-clad eccentricity, cladding ovality and pellet wheatsheafing effects). Calculation of fission gas release and gas bubble swelling is performed by an integrated, highly mechanistic, model [15]. Both intra-granular and inter-granular bubbles are explicitly simulated, with the bubble modelling validated against scanning electron microscopy (SEM) and transmission electron microscopy (TEM) images of irradiated fuel.

The models implemented for the isolated thermalhydraulic subchannel around the fuel rod include: axial distribution of bulk coolant enthalpy; bulk coolant temperature as function of enthalpy; film (rod surface to bulk coolant) temperature drop in both forced convection and nucleate or bulk boiling conditions; crud formation and associated temperature drop.

\section{DEVELOPMENT OF ENIGMA FOR WHOLE CORE ANALYSIS}

Historically, fuel design and licensing was generally performed by evaluating the thermo-mechanical behaviour of the limiting rod in the core with respect to each design criterion. A hypothetical bounding power history was also generally assumed, such that the power versus burnup curve for the limiting rod was an upper bound to all equivalent rod specific curves. This allowed modelling 
only a single rod for each design criterion analysis, and so had the advantage of minimal computation time. However, the results were subject to significant conservatism (thereby limiting plant performance and operational flexibility), the extent of which was often difficult to quantify, and for certain design criteria it was often difficult to prove that the approach taken was limiting.

The alternative of whole core fuel performance modelling, where the thermo-mechanical behaviour of every fuel rod in the core is simulated, is therefore becoming more common. This approach has the advantages of reduced conservatism (and hence more margin to design limits), more easily quantifiable uncertainties, the straightforward identification of limiting rods, and the possibility to introduce more advanced methodologies. The main disadvantage is the large amount of computing power required. However, this disadvantage is becoming less of a barrier as the available computing power inexorably increases. In particular, use of modern clusters, with parallel processing on multiple CPUs, is allowing calculations to be made that were previously not viable $[19,20]$.

The NEXUS code [21] has been developed by NNL to automate whole core fuel performance modelling for an LWR core, using ENIGMA as the underlying fuel performance engine. NEXUS runs on NNL's GEMSTONE high performance computing cluster (comprising 250 nodes, each of which has a dual core CPU and $8 \mathrm{~GB}$ or more of RAM) and utilises 3-D core power distribution data obtained from the output of Studsvik Scandpower's SIMULATE code [8]. NEXUS uses a master-slave approach, whereby a thread running on a 'master' node performs all the initial setup and distributes the ENIGMA calculational workload across accessible GEMSTONE 'slave' nodes. The slaves 'listen' for calls from the master node to begin an ENIGMA calculation. Once a call is made and all the necessary data are passed, the slave performs the ENIGMA calculation and when complete informs the master by returning a success/error code. The master-slave communication utilises the Message Passing Interface (MPI) protocol.

In order to maximise the coupling between NEXUS and ENIGMA (and therefore minimise communication in the form of intermediate files or processes) the ENIGMA source code was re-structured as a FORTRAN subroutine within the NEXUS C++ main program. NEXUS run times were further reduced by suppressing graphical output, minimising ASCII output, and optimising compilation options. A restart capability was also added to ENIGMA such that the thermo-mechanical state of a given rod can be saved at any point in the form of a 'restart file'. This 'restart file' can then be used to restart the analysis from the saved state, facilitating on-line, real time fuel performance surveillance, and branch cases (which could be, for example, a range of different transients from a given initial steady state).

Visualisation of ENIGMA's rod-by-rod fuel performance parameter predictions is possible with a data viewing tool specifically designed to deal with the vast amount of data generated in a full core NEXUS assessment. The visualisation tool generates pin-by-pin 2D maps of user-specified fuel performance parameters on a total rod, rod average, userspecified elevation, maximum local (peak node), or minimum local basis (as appropriate for the parameter of interest). Maps can be of either instantaneous, minimum through-life, or maximum through-life values. An example map of instantaneous rod internal pressure is illustrated in Fig. 2.

Using only 24 nodes on the GEMSTONE cluster, a NEXUS analysis of a 193 assembly (four loop) PWR core of $17 \times 17$ fuel $(\sim 51,000$ fuel rods) takes only $\sim 10$ minutes, compared to more than 24 hours if the individual ENIGMA runs were executed on a desktop PC.

NEXUS analyses have been performed as part of two customer projects: plutonium disposition studies for the UK Nuclear Decommissioning Authority (NDA) [16]; and analysis of an operational event in one of Southern Nuclear Operating Company's PWRs (in co-operation with Studsvik Scandpower) [17]. In the first project NEXUS was used in stand-alone mode, while in the second it was used within the ONUS on-line fuel performance surveillance framework [21].

A NEXUS whole core fuel performance assessment can give the evolution with time (or burnup) of any fuel performance parameter of interest at every axial elevation (where applicable) for every rod in a given core. The parameters of interest include fuel centreline temperature, rod internal pressure, clad hoop strain, stress-corrosion crack length and clad hoop stress. For any given cycle of an operating reactor, a NEXUS analysis can be performed prior to, during, or after the cycle, giving results pertinent to fuel licensing or scenario assessment, on-line surveillance, and (post-operation) analysis of irradiations (including operational manoeuvres), respectively. A pre-cycle analysis can also be performed for suggested cycles of any proposed new reactor.

A basic NEXUS pre-cycle scenario assessment, based on steady-state operation with best estimate input parameters and best estimate models, can be performed with NEXUS as is. More comprehensive assessments, where the effects of transients, input parameter uncertainties, and/or model uncertainties are taken into account can also be performed, but would potentially require multiple NEXUS runs, giving significant cumulative run times and generating large quantities of data. A pre-cycle fuel licensing assessment, which would be equivalent to a fully comprehensive pre-cycle scenario assessment, would be even more challenging. NEXUS and ENIGMA development could make these latter two types of assessment more tractable. Specific improvements would include: automating SIMULATE modelling of the relevant transients (typically start, middle and end of cycle) as branch cases; an automated root mean square (RMS) or Monte Carlo treatment of 


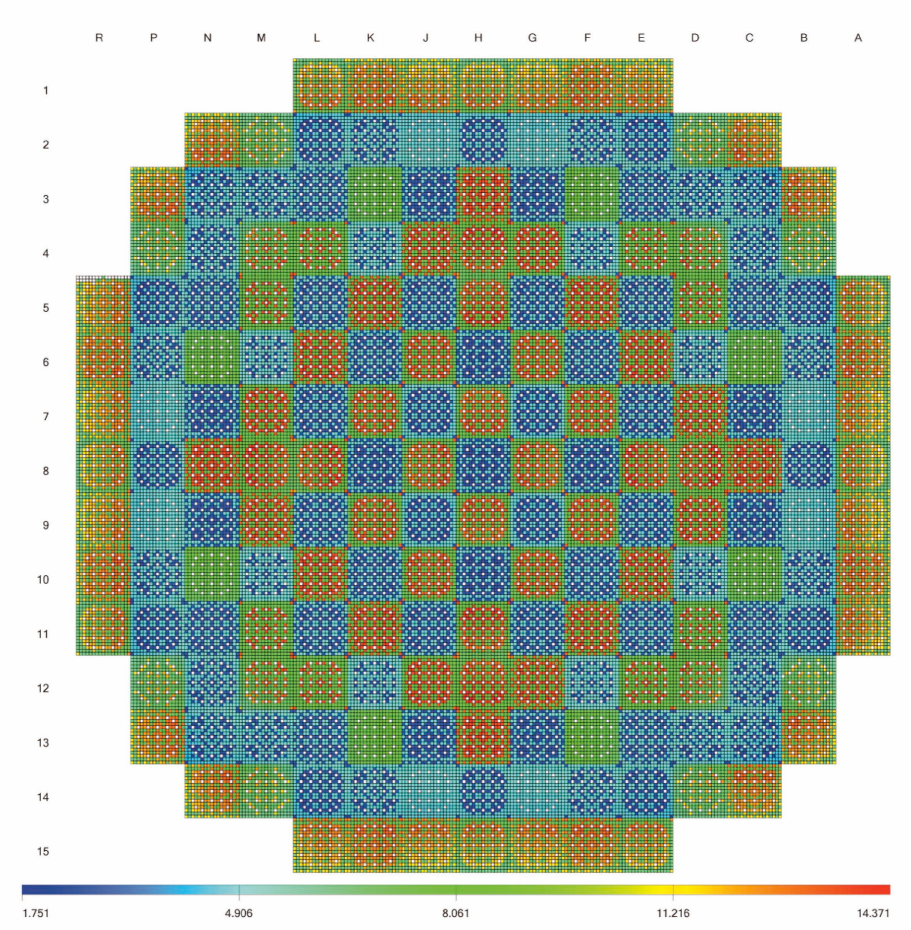

Fig. 2. Sample Map of ENIGMA Predictions from Full Core NEXUS Assessment: Instantaneous Rod Internal Pressure (MPa) for Every Rod in the Core

uncertainties for the limiting rods using ENIGMA; automation in NEXUS of fuel licensing design criteria analysis (coding of methodologies and design limits). This is currently being addressed as part of NNL's Signature Research Programme (an internal R\&D programme whereby a fraction of NNL's profits is fed back into the business to stimulate innovation).

The results from a pre-cycle NEXUS assessment can be used to indicate the margins to the fuel licensing design limits (with the scope and accuracy dependent upon the comprehensiveness of the assessment). Due to the relatively short NEXUS run times, this enables a rapid, heavily automated, fuel rod design assessment by the core designer. Hence, proposed loading patterns which meet the core design limits (on, for example, power peaking) but which would fail one or more fuel rod design limits (on, for example, rod internal pressure) can be identified at an early stage, saving much time and effort in the overall fuel licensing or scenario assessment process. A pre-cycle assessment could also be used to investigate the fuel performance implications of any planned operational manoeuvres during the cycle. This could include a sensitivity study of the effects of varying operational parameters, such as the ramp rate during reactor start-up.

A NEXUS assessment performed during a given cycle allows the comparison of evolving fuel performance parameters against operational (for example, PCI) or licensing (for example, rod internal pressure) limits. This can simply be done at discrete intervals during the cycle (with SIMULATE output files generated for each of these intervals) in a stand-alone manner. Alternatively, NEXUS can be linked with Studsvik Scandpower's CMSOps online surveillance tool (the ONUS framework) to allow continuous, real time monitoring to be performed [21]. Similarly, a sensitivity study for a given planned operational manoeuvre, using the within cycle pre-manoeuvre statepoint as a starting point can be performed. This could, for example, enable the tolerable (with respect to PCI) insertion rates and maximum insertion levels of a given control rod bank during a control rod re-configuration to be determined.

A post-cycle NEXUS assessment allows the fuel performance implications of an event that has occurred to be determined. The event could be either an operational manoeuvre or a fault. If the event was not predicted to lead to any fuel rod failures, a sensitivity study could also be performed to quantify how much more severe the event would have had to have been to cause failure.

With respect to PCI assessments, the maximum local clad hoop stress is a key indicator for predicting the extent of pellet-clad interaction, which is a direct cause of failed fuel. ENIGMA, with its modelling of pellet wheatsheafing, clad ridging, and clad stress concentration 
over radial fuel cracks, is ideally suited to calculating this indicator parameter. Furthermore, ENIGMA has a stresscorrosion crack growth model which can be used to directly predict whether or not PCI failure will occur for a given rod. ENIGMA can also be used to calculate fuel deconditioning and reconditioning rates, conditioned powers, and/or cold fuel-clad gap sizes for use with empirical PCI failure thresholds.

\section{DEVELOPMENT OF ENIGMA FOR DRY STORAGE ASSESSMENTS}

There is an ongoing need for site licence companies and utilities to develop and implement detailed plans for the disposition of spent nuclear fuel, and for regulators to approve these plans. The disposition could include reprocessing, storage (both interim and long-term) and/or final disposal. There are also handling and transport issues associated with these options. With this in mind, NNL have developed ENIGMA for modelling dry storage scenarios (including analysis of the pre-storage drying process). The ENIGMA modelling sits within a broader NNL framework for dry storage simulation which includes: calculation of previous irradiation history using CMS; calculation of the decay heat output, and the actinide, fission product and activation product inventory of the spent fuel using the FISPIN code [22] with nuclear crosssections supplied by CASMO [7]; calculation of clad temperatures during pond cooling, fuel assembly drying and/or storage (in a cask, vault, silo or dry well) using inhouse and commercial thermal analysis / computational fluid dynamics (CFD) codes (including the ANSYS FLUENT software [23]).

The relatively high clad temperatures and high rod internal pressures during drying and dry storage result in permanent creep deformation of the cladding over time. The creep strains can be enhanced in cladding with substantial metal loss due to significant in-pile clad oxidation. The dominant clad failure mechanism during dry storage (at least for zirconium-alloy clad LWR fuel) is therefore creep rupture. Severe embrittlement or delayed hydride cracking as a result of the formation of radial hydrides can also lead to clad failure, and are major concerns. Hence, the primary aim of ENIGMA analysis is to predict whether or not the cladding could fail via these mechanisms. This is done by comparing the clad hoop stresses, clad creep hoop strains and clad temperatures that are predicted during the post-irradiation operations to safety limits, typically 90 or $120 \mathrm{MPa}, 1 \%$, and 370 to $570^{\circ} \mathrm{C}$, respectively [24]. (Thus, radial hydride formation is not modelled explicitly - instead the clad hoop stress is shown to be maintained below a safety limit which precludes the formation of radial hydrides.)

In order to achieve the above, ENIGMA has been developed such that it can model the thermo-mechanical behaviour of a given LWR fuel rod during irradiation, pond cooling, drying, and dry storage (assuming the fuel rod has not failed during irradiation). This involved: (a) incorporating an out-of-pile clad creep model for irradiated Zircaloy-4 (discussed further below); (b) including the ability to simulate annealing out of the clad irradiation damage; (c) writing of additional post-irradiation output; (d) several other minor modifications to allow modelling of post-irradiation conditions, including suppression of clad corrosion modelling, optional use of in-pile or outof-pile clad creep correlations for the post-irradiation operation, optional application of a multiplier to the fission gas diffusion coefficient to facilitate investigating the effects of uncertainties on fission gas release on postirradiation behaviour, and changes to the format of times in the main output to cater for simulated operation over 100 years and more. The ability to vary the rod external pressure from in-reactor conditions (typically $15.5 \mathrm{MPa}$ for a PWR, or 7.5 MPa for a BWR) to pond cooling conditions (atmospheric plus a hydrostatic component), to drying conditions (zero for vacuum drying), to dry storage conditions (typically $\sim 7$ bar [25]) was adapted from the already existing capability for modelling reactor changes.

The suppression of clad corrosion during pond cooling (such that no corrosion is predicted) is consistent with the conditions in spent fuel ponds - that is stagnant water, clad temperatures of $50^{\circ} \mathrm{C}$ or less, and the absence of a fast neutron flux - which mean that corrosion in this environment will generally be negligible (primarily because the corrosion rate is strongly temperature dependent). The exception to this is if the oxidation is exacerbated by dissolved salts or other solutes, but the controls on pond chemistry should preclude this. Thus, the pond storage should cause insignificant additional corrosion to that which has already occurred under irradiation. This is supported by general wet storage experience [26], and, in particular, by the results of the IAEA's BEFAST III coordinated research programme [27].

The suppression of clad corrosion during drying and dry storage (such that no corrosion is predicted) is consistent with the short timescales of the drying procedure, the thermal inertia of the spent fuel and any canister within which it is dried, and the inert gas environment (generally helium or nitrogen) during dry storage (or the low clad temperatures with an air spent fuel cover gas). During storage there will be some residual moisture, but the fuel drying process is designed to limit this to a low level (which is, in any case, required to maintain cask integrity because of pressurisation concerns). This is supported by Peehs and Fleisch [26].

Although ENIGMA continues to model diffusiondriven fission gas and helium release in post-irradiation conditions, the release of gas from the fuel pellets to the rod free volume is negligible due to the lack of fission and the relatively low fuel temperatures compared to those during irradiation. There is, however, the potential of 
fission gas and/or helium release during post-irradiation operations due to: (i) a diffusion enhancement caused by self-irradiation damage (i.e. where alpha particles from transuranic isotope decays either knock gas atoms from their lattice positions or create damage which allows diffusion to occur more readily); (ii) degradation of the fuel matrix by the accumulation of helium; or (iii) fragmentation of the fuel during handling or transport, all of which are not modelled by ENIGMA (although ENIGMA does model the helium accumulation itself, which is due to alpha decay of transuranic isotopes [28]). Given the very long timescales, (i) and (ii) have been studied in some detail in the context of spent fuel geological disposal. In this context, a review by Swedish researchers [29] concluded that both self-irradiation damage and degradation of the fuel matrix due to helium accumulation have negligible effects on gas release. (The former is to be expected given the fact that the alpha particle flux is orders of magnitude lower than the in-pile fission fragment flux.) Hence, the lack of modelling of self-irradiation damage or helium-induced fuel matrix degradation is unimportant. As for fragmentation of the fuel during handling or transport, this is still in theory possible, but operational procedures should preclude its occurrence (except during accidents involving impacts, which are outside the scope of ENIGMA modelling). Thus, the lack of modelling of fragmentation of the fuel during handling or transport is also justified.

The out-of-pile clad creep model for irradiated Zircaloy-4 that was implemented in ENIGMA is based on the creep formulation developed by Bouffioux, Limon et al [30,31], since this is cited by several independent organisations as the best available model for dry storage applications - see, for example, publications by CEA [32], EPRI [33] and CIEMAT [34]. The creep formulation relates hoop strain, $\varepsilon$, to elapsed time, $\mathrm{t}$, via the equation:

$$
\varepsilon=\varepsilon_{0} f \ln (1+v . t)+k f_{k} t
$$

where the first term represents primary (time-dependent) creep and the second term represents secondary (steady state) creep. The multipliers in the primary and secondary creep terms ( $\varepsilon_{0}$ and $\mathrm{k}$, respectively) are each functions of the hoop stress, $\sigma$, and absolute temperature, $T$, as follows:

$$
\varepsilon_{0}=A_{1} \sigma^{n 1} \exp \left(-Q_{1} / T\right)
$$

and

$$
\mathrm{k}=\mathrm{A}_{2}\left(\sinh \left(\mathrm{a}_{0} \cdot \sigma\right)\right)^{\mathrm{n} 2} \exp \left(-\mathrm{Q}_{2} / \mathrm{T}\right)
$$

The $f$ and $f_{k}$ quantities represent irradiation hardening of the primary and secondary creep strains, respectively, and are expressed as functions of the fast neutron dose, $\Phi$, such that:

$$
f=P+(1-P) \exp (-\alpha \Phi)
$$

and

$$
\mathrm{f}_{\mathrm{k}}=\mathrm{S}+(1-\mathrm{S}) \exp (-\beta \Phi)
$$

Overall, the creep law is a function of four independent variables (time, hoop stress, temperature, and fast neutron dose) and contains twelve empirically-derived constants as follows (with hoop strain in \%, time in hours, hoop stress in $\mathrm{MPa}$, temperature in $\mathrm{K}$, and dose in $\mathrm{n} / \mathrm{cm}^{2}$ ): $v=0.13244$; $\mathrm{A}_{1}=20867.2 ; \mathrm{n}_{1}=1.986 ; \mathrm{Q}_{1}=13748 ; \mathrm{A}_{2}=1.386 \times 10^{14} ;$ $\mathrm{n}_{2}=1.715 ; \mathrm{Q}_{2}=27628.7 ; \mathrm{a}_{0}=0.01453 ; \mathrm{P}=0.0879 ; \alpha=$ $5.722 \times 10^{-22} ; \mathrm{S}=0.00305$; and $\beta=3.246 \times 10^{-22}$.

The out-of-pile creep model for irradiated Zircaloy-4 consists of the creep formulation above (after conversion from a hoop strain versus hoop stress form to the generalised strain versus generalised stress form employed in ENIGMA) together with a strain-hardening assumption when applying the creep law under conditions of varying stress and temperature. Experimental tests have shown that the strainhardening approach is applicable to irradiated Zircaloy cladding [35]. The strain-hardening approach works as follows. After a period of straining at one stress and temperature, an effective starting time is determined that would give the same strain value at the new level of stress and temperature. The strain increment for the new step is then calculated using this effective starting time. The effective time calculation requires the creep equation to be inverted numerically, which ENIGMA accomplishes using Newton-Raphson iteration with a starting guess based on first order expansion of the logarithmic term.

ENIGMA dry storage assessments (for storage times of up to 100 years) have been performed for spent $\mathrm{UO}_{2}$ and MOX fuel from both existing PWRs and new build units. Sample output from one of these assessments (3.9 year cooled $\mathrm{UO}_{2}$ fuel irradiated in a commercial PWR to an assembly average burnup of $47 \mathrm{MWd} / \mathrm{kgU}$ ) is illustrated in Fig. 3, where, in this case, drying was ignored. The time is the elapsed time since the start of irradiation. 'Maximum' is with respect to axial variations (not time).

The cladding was modelled as fully annealed during the entire dry storage period (but not during the prior pond cooling). This ensures conservative clad creep predictions (since annealing removes the hardening effects of irradiation damage). This is conservative for three reasons: (a) it assumes full annealing of the irradiation damage; (b) it assumes that the annealing occurs instantaneously at the beginning of dry storage; (c) it ignores the known hardening effect of the hydrogen absorbed by the cladding during the in-pile oxidation process.

The maximum clad (surface) temperature (in red) is between 350 and $370^{\circ} \mathrm{C}$ during irradiation, drops to $50^{\circ} \mathrm{C}$ during pond cooling, and rises to just under $400^{\circ} \mathrm{C}$ at the beginning of dry storage, after which it reduces as the decay heat reduces.

The maximum clad oxide thickness (in green) increases from zero to $53 \mu \mathrm{m}$ during the irradiation and then stays constant at this value during pond cooling and dry storage (since no out-of-pile oxidation is modelled).

The rod internal pressure (in magenta) increases from the fill pressure to $7.0 \mathrm{MPa}$ during the first ramp to power. 

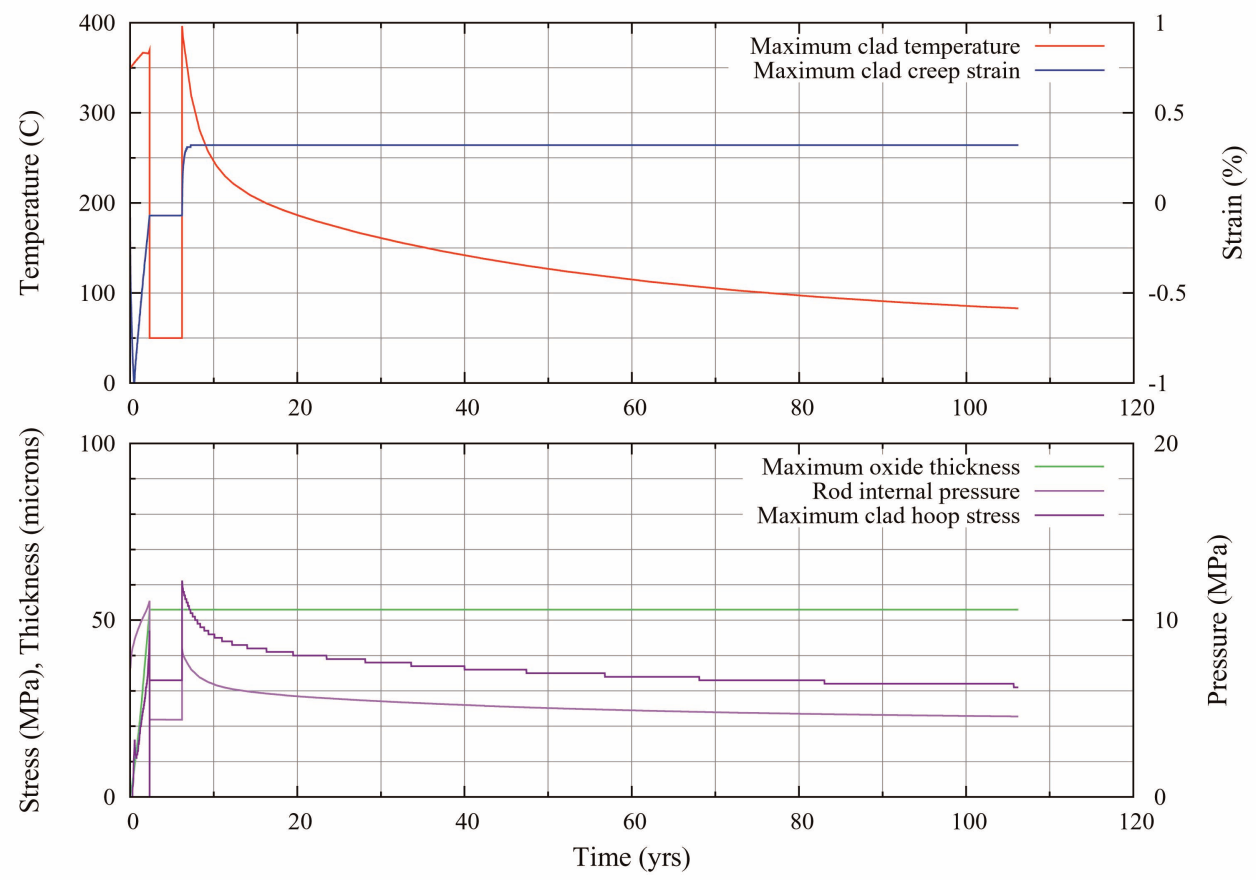

Fig. 3. Sample Output from ENIGMA dry Storage Assessment (3.9 year Cooled $\mathrm{UO}_{2}$ Fuel Irradiated in a Commercial PWR to an Assembly Average Burnup of $47 \mathrm{MWd} / \mathrm{kgU}$ )

The pressure then increases as fission gas release occurs to reach an at-power value of $11.1 \mathrm{MPa}$ by the end of irradiation. The pressure drops to a low value during the end-of-irradiation shutdown and the subsequent pond cooling. It increases sharply during the heat-up at the start of dry storage, then reduces as the gas temperature reduces with time.

The maximum clad hoop stress (in purple) is initially negative (i.e. compressive) in the open fuel-clad gap conditions early in life. The stress then becomes positive (i.e. tensile) as PCMI occurs later in the irradiation. The stress drops during the end-of-irradiation shutdown as PCMI stops and the gas temperature reduces, but immediately increases to $33 \mathrm{MPa}$ in pond cooling conditions when the external pressure is reduced from the reactor coolant pressure to the hydrostatic pressure in the spent fuel pond (conservatively modelled as atmospheric pressure). The stress increases significantly to $61 \mathrm{MPa}$ during the heatup at the start of dry storage (as the gas temperature increases), then decays away as stress is relieved by clad creep.

The maximum clad creep strain (in blue) reduces to negative (i.e. compressive) values during the early period of irradiation due to clad creepdown. There is a change in gradient when PCMI occurs and the strain increases towards positive (i.e. tensile) values. The creep strain reaches a value of $-0.07 \%$ by the end of irradiation, which is unchanged during pond cooling due to the low clad temperatures. The creep strain increases rapidly during the latter stages of the heat-up at the start of dry storage and during the initial period of steady state operation. However, due to the reducing clad temperature, the creep strain levels off to the end of dry storage value of $0.32 \%$ after approximately one year into the 100 year storage period.

\section{SUMMARY}

NNL's version of the ENIGMA fuel performance code for LWR applications is a state-of-the-art tool for simulation of the thermo-mechanical behaviour of LWR fuel. NNL are working with Studsvik Scandpower to allow international access to ENIGMA via licence agreements.

The recent development of ENIGMA has focussed on whole core analysis and dry storage applications. With respect to the former, the NEXUS code has been developed by NNL to automate whole core fuel performance modelling for an LWR core, using ENIGMA as the underlying fuel performance engine. With respect to the latter, ENIGMA has been developed such that it can model the thermomechanical behaviour of a given LWR fuel rod during irradiation, pond cooling, drying, and dry storage.

The whole core analysis and dry storage applications development is part of a broader ENIGMA maintenance 
and development programme at NNL; this broader programme includes validation database updating, model development and capability enhancement (in areas including fuel design and licensing, whole core modelling, spent fuel storage and final disposal applications, fuel surveillance, and analysis of severe transients) and is informed by customer feedback and participation in international programmes (including the OECD Halden Reactor Project [36] and the IAEA FUMEX-III co-ordinated research project [37]). The ENIGMA development programme is itself directed by NNL's Signature Research Programme for internally funded $R \& D$.

\section{ACKNOWLEDGEMENTS}

My colleagues Robert Gregg and Ian Palmer have been instrumental in developing the ENIGMA whole core analysis and dry storage assessment capability.

\section{REFERENCES}

[1] P. A. Jackson, J. A. Turnbull and R. J. White, "A Description of the ENIGMA Fuel Performance Code", Proc. IAEA Technical Committee Meeting on Water Reactor Fuel Element Computer Modelling in Steady-State, Transient and Accident Conditions, Preston, UK, Sep. 19-22, 1988.

[2] P. A. Jackson, J. A. Turnbull and R. J. White, "ENIGMA Fuel Performance Code", Nuclear Energy, 29, 107 (1990).

[ 3 ] W. J. Kilgour, J. A. Turnbull, R. J. White, A. J. Bull, P. A. Jackson and I. D. Palmer, "Capabilities and Validation of the ENIGMA Fuel Performance Code", Proc. ANS/ENS International Topical Meeting on LWR Fuel Performance, Avignon, France, Apr. 21-24, 1991.

[4] J. C. Killeen, "Comparison of the ENIGMA Code with Experimental Data on Thermal Performance, Stable Fission Gas and Iodine Release at High Burn-Up", Proc. IAEA Technical Committee Meeting on Water Reactor Fuel Element Modelling at High Burnup and Its Experimental Support, Windermere, UK, Sep. 19-23, 1994.

[5] G. A. Gates, P. M. A. Cook, P. de Klerk, P. Morris and I. D. Palmer, "Thermal Performance Modelling with the ENIGMA Code", Proc. NEA/CEA Seminar on Thermal Performance of High Burn-Up LWR Fuel, Cadarache, France, Mar. 3-6, 1998.

[6] I. Palmer, G. Rossiter and R. White, "Development and Validation of the ENIGMA Code for MOX Fuel Performance Modelling", Proc. IAEA International Symposium on MOX Fuel Cycle Technologies for Medium and Long Term Deployment: Experience, Advances, Trends, Vienna, Austria, May 17-21, 1999.

[ 7 ] J. Rhodes, K. Smith and D. Lee, "CASMO-5 Development and Applications", Proc. ANS Topical Meeting on Reactor Physics (PHYSOR-2006), Vancouver, Canada, Sep. 10-14, 2006.

[8 ] T. Bahadir and S-Ö Lindahl, "Studsvik's Next Generation Nodal Code SIMULATE-5", Proc. Advances in Nuclear Fuel Management IV (ANFM 2009), Hilton Head Island, South Carolina, USA, Apr. 12-15, 2009.

[9] J. Mullen, C. Brown, I. D. Palmer and P. Morris, "Performance of SBR MOX Fuel in the Callisto Experiment", Proc. TopFuel'97, Manchester, UK, Jun. 9-11, 1997.
[10] R. J. White, S. B. Fisher, P. M. .A. Cook, R. Stratton, C. T. Walker and I. D. Palmer, "Measurement and Analysis of Fission Gas Release from BNFL's SBR MOX fuel”, $J$. Nucl. Mat., 288, 43 (2001).

[11] R. Weston, I. D. Palmer, J. M. Wright, G. D. Rossiter, R. C. Corcoran, T. C. Gilmour, C. T. Walker and S. Bremier, "Progress on SBR MOX Fuel Development", Proc. TopFuel 2001, Stockholm, Sweden, May 27-30, 2001.

[12] S. B. Fisher, R. J. White, P. M. A. Cook, S. Bremier, R. C. Corcoran, R. Stratton, C. T. Walker, P. K. Ivison and I. D. Palmer, "Microstructure of Irradiated SBR MOX Fuel and its Relationship to Fission Gas Release", J. Nucl. Mat., 306, 153 (2002).

[13] M. Barker, P. Cook, R. Weston, G. Dassel, C. Ott, R. Stratton, D. Papaioannou and C. Walker, "Ramp Testing of SBR MOX Fuel", Proc. NEA Seminar on Pellet-Clad Interaction in Water Reactor Fuels, Aix-en-Provence, France, Mar. 9-11, 2004.

[14] M. A. Barker, K. Stephenson and R. Weston. "The Manufacture and Performance of HomogeneousMicrostructure SBR MOX Fuel", Proc. 2007 International LWR Fuel Performance Meeting, San Francisco, California, USA, Sep. 30 - Oct. 3, 2007.

[15] M. A. Barker and C. P. Chatwin, "Fuel Performance Activities at Sellafield Ltd and the UK's National Nuclear Laboratory", Proc. 2008 Water Reactor Fuel Performance Meeting, Seoul, Korea, Oct. 19-23, 2008.

[16] A. Worrall, T. J. Abram, R. W. H. Gregg, K. W. Hesketh, I. D. Palmer, G. D. Rossiter and G. M. Thomas, "Plutonium Utilization Options in Future UK PWRs Using MOX and Inert Matrix Fuels", Proc. Global 2007, Boise, Idaho, USA, Sep. 9-13, 2007.

[17] A. Alapour, R. M. Joyce, A. S. DiGiovine, S. Tarves, N. Patino, A. Worrall, R. Gregg and G. Rossiter, "Robust PCI Monitoring During PWR Operation at Southern Nuclear", Proc. 2010 LWR Fuel Performance/TopFuel/WRFPM, Orlando, Florida, USA, Sep. 26-29, 2010.

[18] G. D. Rossiter, P. M. A. Cook and R. Weston, "Isotopic Modelling Using the ENIGMA-B Fuel Performance Code", Proc. IAEA Technical Committee Meeting on Nuclear Fuel Behaviour Modelling at High Burnup and Its Experimental Support, Windermere, UK, Jun. 19-23, 2000.

[19] R. Gregg, "Whole Core Fuel Performance Modelling", Proc. IAEA Technical Meeting on Fuel Behaviour Modelling Under Normal, Transient and Accident Conditions, and High Burnups, Kendal, UK, Sep. 5-8, 2005.

[20] G. Thouvenin, P. Thévenin, N. Tallet, S. Aunay and S. Lemercier, "High Performance Computing of PWR Simulations with Fuel Code CYRANO3 Running on IBM System Blue Gene/P”, Proc. TopFuel 2009, Paris, France, Sep. 6-10, 2009.

[21] A. Worrall and A. S. DiGiovine, "ONUS: On-line Fuel Performance Surveillance — Linking Studsvik's CMS with UK NNL's ENIGMA-B", Proc. Advances in Nuclear Fuel Management IV (ANFM 2009), Hilton Head Island, South Carolina, USA, Apr. 12-15, 2009.

[22] R. F. Burstall, "FISPIN: A Computer Code for Nuclide Inventory Calculations", ND-R-328R, United Kingdom Atomic Energy Authority (1979).

[23] http://www.ansys.com/Products/Simulation+Technology/ Fluid+Dynamics/ANSYS+FLUENT 
[24] H. G. Kim, Y. H. Jeong and K. T. Kim, "The Effects of Creep and Hydride on Spent Fuel Integrity During Interim Dry Storage", Nucl. Eng. Technol., 42, 249 (2010).

[25] "Final Safety Analysis Report for the HI-STORM 100 Cask System", HI-2002444 Revision 8, Holtec International (2010).

[26] M. Peehs and J. Fleisch, "LWR Spent Fuel Storage Behaviour", J. Nucl. Mat., 137, 190 (1986).

[27] "Further Analysis of Extended Storage of Spent Fuel: Final Report of a Coordinated Research Programme on the Behaviour of Spent Fuel Assemblies during Extended Storage (BEFAST-III), 1991-1996", IAEA-TECDOC944, International Atomic Energy Agency (1997).

[28] J. P. Piron, M. Pelletier and J. Pavageau, "Helium Behaviour in Spent $\mathrm{UO}_{2}$ and MOX Fuels", Proc. NEA Seminar on Fission Gas Behaviour in Water Reactor Fuels, Cadarache, France, Sep. 26-29, 2000.

[29] L. O. Werme, L. H. Johnson, V. M. Oversby, F. King, K. Spahiu, B. Grambow and D. W. Shoesmith, "Spent Fuel Performance Under Repository Conditions: A Model for Use in SR-Can", TR-04-19, Svensk Kärnbränslehantering AB (2004).

[30] P. Bouffioux, S. Leclercq, C. Cappelaere and T. Bredel, "Interim Dry Storage of PWR Spent Fuel Assemblies Development of a Long Term Creep Law to Assess the Fuel Cladding Integrity", Proc. 8th International Conference on Radioactive Waste Management and Environmental Remediation (ICEM'O1), Bruges, Belgium, Sep. 30 - Oct. 4, 2001.

[31] R. Limon, C. Cappelaere, T. Bredel and P. Bouffioux, "A Formulation of the Spent Fuel Cladding Creep Behaviour for Long Term Storage", Proc. ANS International Topical Meeting on LWR Fuel Performance, Park City, Utah, USA, Apr. 10-13, 2000.

[32] C. Ferry, C. Poinssot, P. Lovera and J. M. Gras, "Key Issues from the French R\&D Project on the Long Term Evolution of the Spent Nuclear Fuel in Conditions of Interim Dry Storage", Proc. IAEA International Conference on Storage of Spent Fuel from Power Reactors, Vienna, Austria, Jun. 2-6, 2003.

[33] J. Y. R. Rashid and A. J. Machiels, "Examination of the Creep Rupture Phenomenon and the Development of an Acceptance Criterion for Spent Fuel Dry Storage", Proc. IAEA International Conference on Storage of Spent Fuel from Power Reactors, Vienna, Austria, Jun. 2-6, 2003.

[34] F. Feria and L. E. Herranz, "Review and Adaptation of FRAPCON-3 Creep Model to Dry Storage Conditions", Proc. Global 2009, Paris, France, Sep. 6-11, 2009.

[35] K. Ito, K. Kamimura and Y. Tsukuda, "Evaluation of Irradiation Effect on Spent Fuel Cladding Creep Properties", Proc. 2004 International Meeting on LWR Fuel Performance, Orlando, Florida, USA, Sep. 19-22, 2004.

[36] http://www.ife.no/hrp

[37] J. Killeen, E. Sartori and T. Tverberg, "FUMEX-III: A New IAEA Coordinated Research Project on Fuel Modelling at Extended Burnup", Proc. TopFuel 2009, Paris, France, Sep. 6-10, 2009.

[38] T. J. Abram, "Modelling the Waterside Corrosion of PWR Fuel Rods", Proc. IAEA Technical Committee Meeting on Water Reactor Fuel Element Modelling at High Burnup and Its Experimental Support, Windermere, UK, Sep. 1923, 1994. 